'Facultad de Medicina. Clínica Alemana Universidad del Desarrollo. Santiago, Chile. ${ }^{2}$ Servicio de Neurología,

Departamento de Neurología y Psiquiatría, Facultad de Medicina, Clínica Alemana. Santiago, Chile. ${ }^{3}$ Departamento de Salud Pública y Epidemiología, Universidad de los Andes. Santiago, Chile.

${ }^{a}$ Residente de Neurología.

${ }^{b}$ Magíster (C) Epidemiología, Departamento de Salud Pública y Epidemiología, Universidad de los Andes. Santiago, Chile. 'Médico Veterinario, Magíster en Salud Pública, Magíster en Bioestadística, Magíster en Administración y Gestión en Salud.

dProfesor de Matemática, Doctor en Bioestadística.

No hubo fuentes de apoyo financiero. Los autores no declaran conflictos de interés.

Recibido el 30 de mayo de 2017, aceptado el 10 de octubre de

Correspondencia a: María Pilar Vicuña Pruzzo mariapilarvicuna@gmail.com

\section{Descripción electrofisiológica del síndrome de túnel carpiano según edad en pacientes adultos}

\author{
PILAR VICUÑ $A^{1, \mathrm{a}, \mathrm{b}}$, JUAN FRANCISCO IDIÁQUEZ $Z^{1, \mathrm{a}}$, \\ PAULA JARA ${ }^{2}$, FRANCISCA PINO ${ }^{2}$, MARCELA CÁRCAMO ${ }^{3, c}$, \\ GABRIEL CAVADA ${ }^{3, d}$, RENATO VERDUGO ${ }^{2}$ \\ Electrophysiological severity \\ of carpal tunnel syndrome according \\ to age in adult patients
}

Background: Carpal tunnel syndrome (CTS) represents $90 \%$ of entrapment neuropathies. Severity may be greater in older patients. Aim: To describe the electrophysiological findings in adult patients with CTS and determine if severity is related to age. Material and Methods: Descriptive and retrospective study of electrophysiological findings in patients over 18 years of age with clinical suspicion of CTS, studied between January 2011 and December 2015. Neurophysiological severity was classified in 3 grades, comparing them by age, gender and laterality. Results: Of 1156 patients subjected to electrophysiological studies due to a clinical suspicion of CTS, 690 (60\%) had electrophysiological features of the disease. In 274 patients (24\%) the compromise was mild, in 162 (14\%) it was moderate and in $254(22 \%)$ it was severe. There was a positive association between age and $C T S$ severity $(p<0.01)$. Severity was significantly greater in males than females $(p<0.01)$. Bilateral CTS was present in 471 patients $(68 \%)$, which was associated with increased age and severity $(p<0.01)$. Conclusions: Electrophysiological severity in CTS increases with age. Other factors associated with higher severity are male gender and bilateral disease.

(Rev Med Chile 2017; 145: 1252-1258)

Key words: Carpal Tunnel Syndrome; Electromyography; Median Neuropathy.
$\mathrm{E}$ 1 síndrome del túnel carpiano (STC) es la mononeuropatía por atrapamiento más frecuente, representando el $90 \%$ de los casos $^{1}$. Se estima una prevalencia clínica de 3,8\% en la población general, disminuyendo a $2,7 \%$ cuando se confirma electrofisiológicamente, con una incidencia que puede llegar hasta 276/100.000 habitantes por año ${ }^{2,3}$. Las mujeres duplican la prevalencia de los hombres, y la edad de presentación más frecuente es entre los 55 y 60 años ${ }^{4,5}$.

La literatura internacional describe un aumen- to progresivo del compromiso electrofisiológico a medida que aumenta la edad, identificándose como un factor de riesgo independiente para el desarrollo de STC $4,6-14$. Los adultos mayores de 65 años tienen una mayor prevalencia de STC grave, lo que se correlaciona clínicamente con atrofia de la eminencia tenar ${ }^{8,14-15}$. La presentación bilateral ocurre en 50 a $60 \%$ de los casos, aumentando esta proporción a mayor edad, duración e intensidad de los síntomas ${ }^{6,15-16}$.

En cuanto al sexo, algunos estudios han de- 
mostrado un mayor compromiso en hombres que mujeres en todas las edades, sin embargo, la literatura aún no avala este hecho ${ }^{4,7,17}$.

Aunque no se ha logrado establecer un criterio estándar para el diagnóstico de STC, Rempel et al. concluyeron que la combinación de los síntomas característicos y las alteraciones electrofisiológicas eran el método más preciso para poder establecer el diagnóstico para estudios epidemiológicos ${ }^{18}$.

En las últimas décadas se han desarrollado diversas escalas clínicas, electrofisiológicas y combinación de ambas, demostrando una correlación estadísticamente significativa entre la clínica y el compromiso electrofisiológico, destacando la escala de Padua et al (1997) y la de Bland (2000) ${ }^{19,20}$.

El STC representa un importante tema de salud pública a nivel nacional, debido a su elevada prevalencia en población laboralmente activa, con una alta morbilidad asociada a ausentismo laboral ${ }^{21}$. Se suman los altos costos relacionados al tratamiento quirúrgico en casos seleccionados, en donde los resultados no siempre son satisfactorios ${ }^{1}$. El estudio electrofisiológico permite confirmar el diagnóstico de STC, siendo además, un marcador indirecto del compromiso clínico ${ }^{6}$. Cabe destacar que en Chile no hay estudios epidemiológicos ni electrofisiológicos de STC.

El objetivo de este estudio es caracterizar los hallazgos neurofisiológicos en pacientes con sospecha de STC en un centro de salud terciario en Santiago de Chile, describiendo el compromiso electrofisiológico según edad. Complementariamente, se evaluará el compromiso de STC según sexo y lateralidad. Por último, se determinará la concordancia entre el diagnóstico realizado por el neurofisiólogo y el diagnóstico de STC según la clasificación electrofisiológica.

\section{Material y Método}

\section{Tipo de estudio}

Estudio descriptivo, observacional y retrospectivo, diseño de serie de casos.

\section{Sujetos del estudio}

Se incluyeron todos los pacientes mayores de 18 años derivados por sospecha clínica de STC a la unidad de Neurofisiología de Clínica Alemana de Santiago (CAS), entre enero de 2011 y diciembre de 2015, que tuviesen evaluación neurofisiológica sensitiva y motora de los nervios mediano y cubital en ambas extremidades superiores. Según datos aportados en la orden de derivación, se excluyeron pacientes con antecedentes de cirugía por STC y a pacientes con otra patología neurológica asociada, como radiculopatías cervicales, polineuropatías o plexopatías, para evitar factores de confusión en el diagnóstico neurofisiológico de STC o en la graduación de su compromiso.

\section{Instrumentos y operadores del estudio}

Todos los estudios se realizaron en el Laboratorio de Neurofisiología de CAS, lugar con climatización programada entre 23 y $25^{\circ} \mathrm{C}$. Se utilizaron los electromiógrafos Synergy On Nicolet EDX (Natus Medical Inc. USA). Los procedimientos fueron realizados por cuatro neurofisiólogos, utilizando los criterios diagnósticos electrofisiológicos para STC de la American Association of Neuromuscular and Electrodiagnostic Medicine (AANEM) ${ }^{22}$ y los rangos de normalidad de la conducción nerviosa motora y sensitiva según lo descrito en la literatura $^{23}$.

Para la clasificación del compromiso electrofisiológico del STC se utilizó la escala de Bland. En esta se mide la conducción nerviosa sensitiva y motora del nervio mediano, incluyendo latencia distal, velocidad de conducción y amplitud de potencial de acción, clasificando el compromiso en seis grados de forma ascendente. Se agregaron valores de corte arbitrarios cuando la escala no los mencionaba explícitamente (ver Anexo 1) ${ }^{20}$.

\section{Recolección de datos}

La información se extrajo de los informes electrofisiológicos disponibles en CD, tabulándose de forma anonimizada los datos de ambas extremidades superiores para cada paciente.

Para la recolección y manejo de datos se obtuvo apoyo del Departamento de Informática de CAS y se utilizó la herramienta electrónica para captura de datos REDCap ${ }^{24}$.

\section{Análisis de datos}

El diagnóstico de STC se definió por grado $\geq 1$ en la escala de Bland, no por el diagnóstico del operador. Para simplificar la interpretación clínica y poder comparar con estudios internacionales, se agruparon de forma arbitraria en 3: STC leve (grado 1 y 2), STC moderado (grado 3 ) y STC grave (grado 4, 5 y 6). Cada paciente fue clasificado basándose en la extremidad superior 
con mayor severidad electrofisiológica según la escala de Bland.

\section{Estadística}

La descripción de las variables continuas se realizó mediante promedio y desviación estándar, mientras que las variables categóricas fueron descritas en frecuencias absolutas y porcentajes. Las variables continuas fueron comparadas mediante el test $\mathrm{t}$-Student o ANOVA y las variables categóricas fueron comparadas mediante el test exacto de Fisher.

La respuesta de compromiso fue asociada con edad y sexo a través de un modelo de regresión logística ordinal. La lateralidad fue asociada con gravedad, sexo y edad a través de un modelo de regresión logística binaria. Las asociaciones encontradas a través de los modelos logísticos fueron expresadas mediante Odds Ratios y su respectivo intervalo de confianza de $95 \%$.

La concordancia entre el diagnóstico realizado por el neurofisiólogo y la clasificación electrofisiológica ( $\geq$ grado 1 en la escala de Bland), se evaluó a través del índice Kappa.

Se utilizó una significación de 5\%. Los datos fueron procesados en el programa estadístico STATA versión 14.0.

\section{Consideraciones éticas}

Estudio revisado y aprobado por el Departamento Científico Docente y el Comité de Ética de CAS. Se cumplieron las normas éticas exigidas internacionalmente.

\section{Resultados}

De un total de 1.187 pacientes, se excluyeron 11 por cirugía previa de STC y 20 por STC asociado a otro diagnóstico neurológico, quedando finalmente 1.156 pacientes con sospecha clínica de STC, de los cuales 897 (77,6\%) fueron mujeres y $259(22,4 \%)$ fueron hombres. La edad mínima fue de 18 años y la máxima de 92 años. La edad promedio fue de 50,1 $( \pm 14,3)$ años; en mujeres fue de 49,5 ( $\pm 14,1)$ años y en hombres de 52,1 $( \pm 14,8)$ años, siendo esta diferencia estadísticamente significativa $(\mathrm{p}=0,012)$.

En $690(59,7 \%)$ pacientes se encontraron hallazgos electrofisiológicos sugerentes de STC, clasificándose $274(23,7 \%)$ en leve, 162 (14\%) en moderado y $254(22 \%)$ en STC grave.
Tabla 1. Grado de compromiso electrofisiológico de STC según sexo

\begin{tabular}{|lcc|}
\hline Severidad STC & $\begin{array}{c}\text { Masculino } \\
\mathbf{n ~ ( \% )}\end{array}$ & $\begin{array}{c}\text { Femenino } \\
\mathbf{n}(\%)\end{array}$ \\
\hline Sin STC & $105(40,5)$ & $361(40,3)$ \\
Leve & $45(17,4)$ & $229(25,5)$ \\
\hline Moderado & $42(16,2)$ & $120(13,4)$ \\
Grave & $67(25,9)$ & $187(20,9)$ \\
\hline
\end{tabular}

Al explorar, se encontró una asociación estadísticamente significativa $(\mathrm{p}=0,028)$ entre intensidad del compromiso de STC y sexo (Tabla 1). Ambos sexos presentaron frecuencias similares de estudios negativos para STC, cercanas a $40 \%$. Dentro de los estudios con hallazgos de STC, la mayoría de los hombres fue clasificado como STC grave $(25,9 \%)$, mientras que la mayoría de las mujeres lo fue como STC leve $(25,5 \%)$.

Se evidencia una gradiente epidemiológica ascendente entre la edad y el mayor compromiso de STC $(p=0,0000)$ (Figura 1). Los pacientes sin hallazgos de STC fueron en promedio los más jóvenes, observándose un aumento progresivo en el promedio de edad desde los STC leves hasta los graves, alcanzando estos últimos un promedio de 58,5 años $( \pm 15,0)$. Al evaluar esta gradiente en términos de un Odds Ratio, se encontró que por cada año de aumento en edad, el riesgo de escalar en el compromiso fue de $5,7 \%(\mathrm{OR}=1,057$, IC 95\% 1,048-1,066) $(\mathrm{p}=0,000)$.

Hubo $471(68,3 \%)$ pacientes con STC que presentaron hallazgos bilaterales, sin haber diferencias estadísticamente significativas por sexo $(\mathrm{p}=0,922)$. El promedio de edad para los hallazgos unilaterales fue de 50,2 años $( \pm 12,6)$, mientras que para los bilaterales fue de 55,7 años $( \pm 14,1)$, con diferencias estadísticamente significativas entre ambos $(\mathrm{p}=0,000)$. Además, se evidenció que los hallazgos bilaterales aumentaban a medida que aumentaba el compromiso $(\mathrm{p}=0,000)$ (Tabla 2$)$. De este modo, la mayoría de los STC unilaterales presentaron un grado de compromiso leve, mientras que la mayoría de los bilaterales fueron graves. Se encontró una mayor probabilidad de presentar STC bilateral en casos de STC moderado, grave y a mayor edad, con diferencias estadísticamente significativas $(\mathrm{p}=0,0000)$. La mayor asociación 


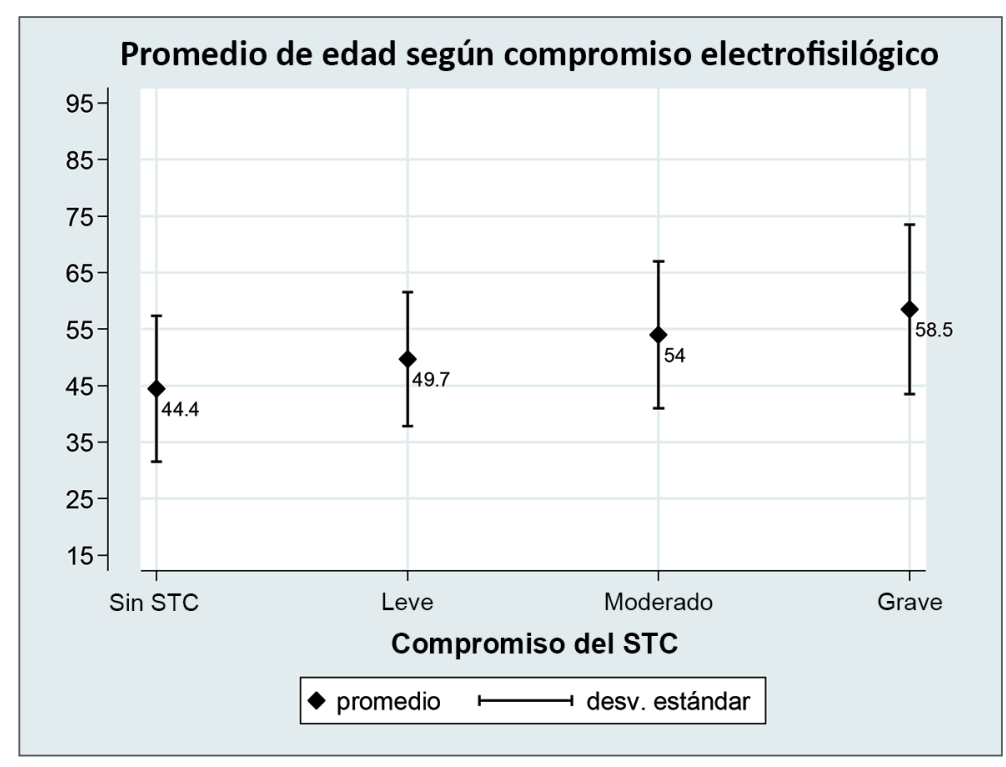

Figura 1. Grado de compromiso electrofisiológica de STC según edad.
Tabla 2. Grado de compromiso electrofisiológico de STC según lateralidad

\begin{tabular}{|lcc|}
\hline $\begin{array}{c}\text { Compromiso } \\
\text { STC }\end{array}$ & $\begin{array}{c}\text { Unilateral } \\
\mathbf{n ~ ( \% )}\end{array}$ & $\begin{array}{c}\text { Bilateral } \\
\mathbf{n}(\%)\end{array}$ \\
\hline Leve & $143(65,3)$ & $131(27,8)$ \\
\hline Moderado & $20(9,1)$ & $142(30,2)$ \\
\hline Grave & $56(25,6)$ & $198(42,0)$ \\
\hline
\end{tabular}

Tabla 3. Riesgo de presentar STC bilateral según compromiso, ajustado por edad y sexo

\begin{tabular}{|lccc|}
\hline STC bilateral & OR & IC 95\% & p-value \\
\hline Leve & 1,00 & - & - \\
\hline Moderado & 7,42 & $4,36-12,62$ & 0,000 \\
\hline Grave & 3,37 & $2,27-5,00$ & 0,000 \\
\hline Edad & 1,02 & $1,01-1,04$ & 0,001 \\
\hline Sexo & 1,27 & $0,83-1,94$ & 0,268 \\
\hline
\end{tabular}

fue con STC moderado, donde fue 7,4 veces más probable que los hallazgos sean bilaterales al compararlo con un STC leve. Por otro lado, el sexo no fue un factor de riesgo para STC bilateral.

Complementariamente, se evaluó la concor- dancia entre el diagnóstico de STC hecho por el neurofisiólogo y el diagnóstico según la clasificación electrofisiológica, siendo de $91,84 \%$ (índice kappa $=0,8 ; \mathrm{p}=0,0000)$, demostrando una alta concordancia entre ambos.

\section{Discusión}

Este estudio describe el perfil epidemiológico y electrofisiológico de pacientes con STC que consultan a un centro terciario de salud. Al igual que en la literatura internacional, gran parte de las derivaciones por sospecha clínica de STC fueron mujeres. La edad promedio de 50,1 años es similar al estudio de Bland, 2000, (51 años) y algo menor que en otros estudios internacionales, pudiendo deberse a la utilización de criterios de inclusión más amplios e inespecíficos ${ }^{19,20}$.

Un elevado porcentaje de pacientes (40,3\%) obtuvo un estudio electrofisiológico negativo, lo cual se explica en parte debido a una sensibilidad de $80-90 \%$ de la técnica, y por otro lado, debido a la derivación de pacientes con sospecha de STC realizada por médicos generales y especialistas de diversas áreas, no solo especialistas neurólogos ${ }^{25}$.

El aumento progresivo del compromiso según edad fue concordante con la literatura interna- 
cional. Se necesitan estudios de cohorte para determinar causalidad, sin embargo, se plantea que pudiese haber una progresión más rápida en adultos mayores, $y$, por otro lado, que fuese un proceso crónico, lento y progresivo que comienza a edades tempranas 6 . Apoya a esto último el hecho que la mayoría presentaron STC bilateral, asociándose a mayor edad y mayor compromiso que los casos unilaterales.

Pese que aún hay discusión en la literatura sobre la relación entre compromiso del STC y sexo, los resultados avalan la teoría que los hombres presentarían casos más graves que las mujeres ${ }^{4}$.

La principal fortaleza del estudio es tener un gran número de pacientes, la ausencia de pérdida de datos y la objetividad de las variables medidas para poder realizar la clasificación electrofisiológica. Como se mencionó previamente, varios estudios han demostrado una correlación entre el compromiso clínico y el deterioro de los hallazgos electrofisiológicos, por lo cual los resultados de este estudio aportan evidencia objetiva en la evaluación del compromiso del STC ${ }^{26}$.

Los datos evidencian un aumento del compromiso electrofisiológico del STC a mayor edad; sin embargo, no es posible determinar la asociación entre ambas variables al no tener sujetos controles pareados por edad. Estos cambios pudiesen ser atribuidos al envejecimiento normal, donde se ha demostrado un aumento en las latencias distales y una disminución en las velocidades de conducción tanto sensitivas como motoras, además de un aumento en la variabilidad de los resultados ${ }^{27,28}$. De todas formas, estudios internacionales han demostrado que en pacientes de mayor edad con STC, el aumento en la latencia distal y la disminución en la velocidad de conducción del nervio mediano son significativamente mayores que en sujetos normales sin STC de la misma edad ${ }^{29-31}$. Al ser un estudio retrospectivo, no permite el seguimiento de los pacientes para medir la evolución electrofisiológica del STC con el paso de los años, para lo cual sería adecuado realizar un estudio de cohorte.

Dado el diseño del estudio, no se dispone de otros antecedentes clínicos de los pacientes, incluyendo factores de riesgo que pudiesen afectar la clasificación del compromiso del STC. En una publicación reciente se evidenció que el grado de lesión desmielinizante y axonal fue mayor en pacientes con STC asociado a diabetes mellitus, artritis reumatoide o hipotiroidismo que en pacientes con STC idiopático ${ }^{32}$.

Cada paciente fue evaluado por uno de los cuatro operadores sin determinar concordancia entre ellos, pudiendo existir un sesgo operador dependiente, sin embargo, hay estudios que han demostrado una alta confiabilidad en las mediciones del nervio mediano en manos de electromiografistas con experiencia ${ }^{33}$. Los operadores son neurólogos especialistas en electrofisiología de nervio periférico, disminuyendo la variabilidad interoperador y la posibilidad de error. Como fue mencionado en la metodología, el diagnóstico se basó en los hallazgos electrofisiológicos y no en el diagnóstico del operador.

Existe sesgo de selección, ya que son pacientes referidos a un centro terciario privado de alto estrato socioeconómico en nuestro país, otorgando mayor acceso y oportunidad para estudiarse que en otros sectores. Esto permite realizar un diagnóstico precoz, aumentando la cantidad de casos clínicamente leves que son estudiados y tener un mejor control de las patologías asociadas a STC. Además, pueden optar a un tratamiento médico temprano disminuyendo el compromiso de la patología en algunos casos. Por otro lado, los pacientes que acuden a CAS, en general, no realizan actividades laborales que requieran de trabajo manual extremo o muy repetitivo, minimizando a este grupo de la población con mayor riesgo de desarrollar STC ${ }^{34}$. Destaca el bajo número de pacientes excluidos por cirugía previa de STC, esto pudiese ser explicado por las características de la población estudiada, o bien por falta de información en el diagnóstico de derivación.

El haber utilizado una escala empírica para la clasificación del compromiso de STC agrupando los 6 estadios de Bland en 3 grandes grupos (leve, moderado y grave), permite acercarse más a la práctica clínica habitual y realizar comparaciones con estudios internacionales que han utilizado métodos similares ${ }^{6}$. Se debe recordar que esta escala permite reconocer el compromiso de la neuropatía del nervio mediano en la muñeca, por lo cual es necesario complementar siempre con la clínica del paciente ${ }^{35}$.

Es de suma importancia mencionar que la ausencia de hallazgos electrofisiológicos no descarta un STC, por lo cual el diagnóstico podría estar subestimado, sin embargo, la combinación de la sospecha clínica asociada a hallazgos elec- 
trofisiológicos de STC representa el mayor nivel de precisión diagnóstica ${ }^{2,28}$. Cabe destacar que no se incluyeron pacientes asintomáticos, evitando falsos positivos.

Esta investigación aporta a la literatura nacional un mayor conocimiento sobre el STC que hasta ahora no había sido estudiado en nuestro país. Se puede concluir que a mayor edad, mayor es el compromiso electrofisiológico del STC. Se asociaron también a mayor compromiso el sexo masculino y la presencia de hallazgos bilaterales.

Agradecimientos: Se agradece a la Unidad de Investigación y Ensayos Clínicos, Departamento Científico Docente de Clínica Alemana de Santiago, por la confección de la base de datos REDCap, especialmente al Dr. Cristóbal Carvajal y Cristopher Gumera.

\section{Anexo 1. Escala neurofisiológica de compromiso de síndrome del túnel carpiano*a}

\section{- Grado 0: Sin evidencias de STC}

- Grado 1: STC muy leve

- Detectado por diferencia de latencia sensitiva distal palma- muñeca cubital y mediano (LC-LM) >0,5 ms

- Grado 2: STC leve

- Velocidad de conducción sensitiva (VCS) muñeca $<40 \mathrm{~m} / \mathrm{s}$

- Latencia motora distal (LMD) $<4,5 \mathrm{~ms}$

- Grado 3: STC moderado

- Latencia motora distal (LMD) $>4,5 \mathrm{~ms}$ y $<6,5 \mathrm{~ms}$

- Potencial de acción sensitivo (SNAP) conservado

- Grado 4: STC grave

- Latencia motora distal $>4,5 \mathrm{~ms}$ y $<6,5 \mathrm{~ms}$

- Potencial de acción sensitivo (SNAP) ausente

- Grado 5: STC muy grave

- Latencia motora distal (LMD) > 6,5 ms

- Amplitud potencial motor (CMAP) $>0,2 \mathrm{mV}$

- Grado 6: STC extremadamente grave

- Amplitud potencial motor (CMAP) $<0,2 \mathrm{mV}$

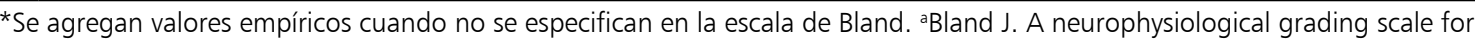
carpal tunnel syndrome. Muscle Nerve 2000; 23 (8): 1280-3.

\section{Referencias}

1. Ibrahim I, Khan W, Goddard N, Smitham P. Carpal Tunnel Syndrome: A Review of the Recent Literature. Open Orthop J 2012; 6 (1): 69-76.

2. Atroshi I, Gummesson C, Johnsson R, Ornstein E, Ranstam J, Rosen I. Prevalence of Carpal Tunnel Syndrome in a General Population. JAMA 1999; 282 (2): 153-8.

3. Mondelli M, Giannini F, Giacchi M. Carpal tunnel syndrome incidence in a general population. Neurology 2002; 58 (2): 289-94.

4. Bland J, Rudolfer S. Clinical surveillance of carpal tunnel syndrome in two areas of the United Kingdom, 19912001. J Neurol, Neurosurg Psychiatry 2003; 74 (12):
1674-9.

5. Gelfman R, Melton L, Yawn B, Wollan P, Amadio P, Stevens J. Long-term trends in carpal tunnel syndrome. Neurology 2009; 72 (1): 33-41.

6. Becker J, Scalco R, Pietroski F, Celli L, Gomes I. Is carpal tunnel syndrome a slow, chronic, progressive nerve entrapment?. Clin Neurophysiol 2014; 125 (3): 642-6.

7. Becker J, Nora D, Gomes I, Stringari F, Seitensus R, Panosso J, et al. An evaluation of gender, obesity, age and diabetes mellitus as risk factors for carpal tunnel syndrome. Clin Neurophysiol 2002; 113 (9): 1429-34.

8. Lam N, Thurston A. Association of obesity, gender, age and ocupation with carpal tunnel syndrome. Aust N Z J Surg 1998; 68 (3): 190-3. 
9. Komurcu H, Kilic S, Anlar O. Relationship of Age, Body Mass Index, Wrist and Waist Circumferences to Carpal Tunnel Syndrome Severity. Neurol Med Chir (Tokyo) 2014; 54 (5): 395-400.

10. Kouyoumdjian J, Zanetta D, Morita M. Evaluation of age, body mass index, and wrist index as risk factors for carpal tunnel syndrome severity. Muscle Nerve 2002; 25 (1): 93-7.

11. Werner R. Electrodiagnostic Evaluation of Carpal Tunnel Syndrome and Ulnar Neuropathies. PM R 2013; 5 (5): S14-S21.

12. Kanatani T, Nagura I, Kurosaka M, Kokubu T, Sumi M. Electrophysiological Assessment of Carpal Tunnel Syndrome in Elderly Patients: One-Year Follow-Up Study. J Hand Surg Am 2014; 39 (11): 2188-91.

13. Blumenthal S, Herskovitz S, Verghese J. Carpal tunnel syndrome in older adults. Muscle Nerve 2006; 34 (1): 78-83.

14. Seror P. Carpal tunnel syndrome in the elderly. "Beware of severe cases". Ann Chir Main Memb Super 1991; 10 (3): 217-25.

15. Hoogstins C, Becker S, Ring D. Contralateral electrodiagnosis in patients with abnormal median distal sensory latency. Hand 2013; 8 (4): 434-8.

16. Michelsen $H$, Posner M. Medical history of carpal tunnel syndrome. Hand Clin 2002; 18 (2): 257-68.

17. Moghtaderi A, Izadi S, Sharafadinzadeh N. An evaluation of gender, body mass index, wrist circumference and wrist ratio as independent risk factors for carpal tunnel syndrome. Acta Neurol Scand 2005; 112 (6): 375-9.

18. Rempel D, Evanoff B, Amadio P, de Krom M, Franklin G, Franzblau A, et al. Consensus criteria for the classification of carpal tunnel syndrome in epidemiologic studies. Am J Public Health 1998; 88 (10): 144751.

19. Padua L, Lo Monaco M, Padua R, Gregori B, Tonali P. Neurophysiological classification of carpal tunnel syndrome: assessment of 600 symptomatic hands. Italian J Neurol Sci 1997; 18 (3): 145-50.

20. Bland J. A neurophysiological grading scale for carpal tunnel syndrome. Muscle Nerve 2000; 23 (8): 1280-3.

21. Aroori S, Spence R. Carpal tunnel syndrome. Ulster Med J 2008; 77 (1): 6-17.

22. American Association of Electrodiagnostic Medicine, American Academy of Neurology, and American Academy of Physical Medicine and Rehabilitation. Practice parameter for electrodiagnostic studies in carpal tunnel syndrome: summary statement. Muscle Nerve 2002; 25 : 918-22.

23. Kimura J. Electrodiagnosis in diseases of nerve and muscle: principles and practice. Oxford: Oxford University Press; 2013.

24. Harris PA, Taylor R, Thielke R, Payne J, González N, Conde JG. Research electronic data capture (REDCap) A metadata-driven methodology and workflow process for providing translational research informatics support. J Biomed Inform 2009; 42 (2): 377-81.

25. Jablecki CK, Andary MT, Floeter MK, Miller RG, Quartly CA, Vennix MJ, et al. Practice parameter: electrodiagnostic studies in carpal tunnel syndrome. Report of the American Association of Electrodiagnostic Medicine, American Academy of Neurology, and the American Academy of Physical Medicine and Rehabilitation. Neurology 2002; 58: 1589-92.

26. Srikanteswara P, Cheluvaiah J, Agadi J, Nagara K. The Relationship between Nerve Conduction Study and Clinical Grading of CTS. J Clin Diagn Res 2016; 10 (7): 13-8

27. Hennessey WJ, Falco FJE, Braddom RL, Goldberg G. The influence of age on distal latency comparisons in carpal tunnel syndrome. Muscle Nerve 1994; 17: 121517.

28. Verdú E, Ceballos D, Vilches J, Navarro X. Influence of aging on peripheral nerve function and regeneration. J Peripher Nerv Syst 2000; 5: 191-208.

29. Bodofsky E, Campellone J, Wu K, Greenberg W. Age and the severity of carpal tunnel syndrome. Electromyogr Clin Neurophysiol 2004; 44 (4): 195-9.

30. Kouyoumdjiam J. Carpal tunnel syndrome: age, nerve conduction severity and duration of symptomatology. Arq Neuropsiquiatr 1999; 57 (2B): 382-6.

31. Srikanteswara P, Cheluvaiah J, Agadi J, Nagaraj K. The Relationship between Nerve Conduction Study and Clinical Grading of Carpal Tunnel Syndrome. J Clin Diagn Res 2016; 10 (7):13-8.

32. Solmaz V, Yavuz S, Inanir A, Aksoy D, Pektas E, Tekatas $\mathrm{A}$, et al. Investigation of Nerve Conduction Studies of Carpal Tunnel Syndrome Cases With Different Risk Factors: An Electrodiagnostic Study. J Clin Neurophysiol 2017; 34: 139-43.

33. Chaudhry V, Cornblath DR, Mellits ED, et al. Inter- and intra-examiner reliability of nerve conduction measurements in normal subjects. Ann Neurol 1991; 30: 841-3.

34. Kozak A, Schedlbauer G, Wirth T, Euler U, Westermann C, Nienhaus. Association between work-related biomechanical risk factors and the occurrence of carpal tunnel syndrome: an overview of systematic reviews and a meta-analysis of current research. BMC Musculoskelet Disord 2015; 16: 231-49.

35. Watson J. The electrodiagnostic approach to carpal tunnel syndrome. Neurol Clin 2012; 30: 457-78. 